\title{
La colegialidad docente en la escuela primaria mexicana: Análisis de sus prácticas
}

\section{Teaching Collegiality in Mexican Primary School: An Analysis of Its Practices}

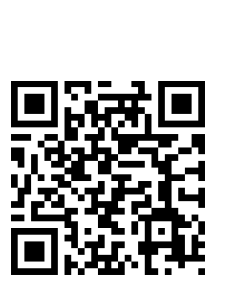

\author{
Efrén Martínez-Cruz' \\ Secretaría de Educación Pública de Hidalgo \\ Pachuca, México \\ efrac5@hotmail.com \\ Tiburcio Moreno-Olivos² \\ Universidad Autónoma Metropolitana-Cuajimalpa \\ Ciudad de México \\ tmoreno@correo.cua.uam.mx
}

Recibido 2 de noviembre de 2015 • Corregido 14 de febrero de 2016 • Aceptado 12 de abril de 2017

Resumen: La colegialidad docente en la escuela primaria mexicana se desarrolla influenciada por una
dinámica global que conforma una cultura escolar compleja, con prácticas educativas singulares que se
han ido sedimentando a través del tiempo. Se realizó una investigación cualitativa de corte etnográfico
mediante la modalidad de estudio de casos. El caso estuvo conformado por 13 profesionales a cargo
de la dirección de educación primaria. Las técnicas para la recogida de los datos fueron la observación
participante, la entrevista semiestructurada y el análisis de documentos. Mediante un proceso de
investigación inductivo se construyeron las categorías de análisis empíricas, para finalmente producir
un texto interpretativo que identifica algunas de las prácticas de colaboración docente que en el
artículo se describen y analizan. Entre los hallazgos principales se identificó que lo que impulsa al
profesorado para colaborar con otras personas tiene que ver con resolver situaciones prácticas e
inmediatas con oportunidad, para que la escuela siga funcionando con "cierta normalidad". También
se pudo constatar que el tiempo para la colegialidad docente no está previsto, por lo que a menudo
el profesorado consume el tiempo del recreo de sus estudiantes, incluso el personal directivo amplía
ese tiempo para desarrollar diferentes reuniones relacionadas con ofrecer información de diverso tipo.

Palabras claves: Colaboración docente; dirección escolar; cultura escolar; prácticas educativas; educación primaria.

\footnotetext{
${ }^{1}$ Licenciado en Educación Primaria, Licenciado en Ciencias Sociales, Maestro en Pedagogía, Doctor en Ciencias de la Educación. Actualmente profesor de educación primaria. Desde 1985 me he desempeñado como profesor frente a grupo, asesor técnico pedagógico, supervisor escolar y director de escuela en educación primaria. Alternativamente 10 años como catedrático en la Escuela Normal del Estado de Hidalgo, 5 años como asesor académico en la Universidad Pedagógica Nacional, unidad 131 de Hidalgo. Poseo en coautoría con el Dr. Tiburcio Moreno Olivos una publicación en la revista Revalue en el año 2015.

${ }^{2}$ Licenciado en Pedagogía por la Universidad Veracruzana (México) y Doctor en Pedagogía por la Universidad de Murcia (España). Actualmente es profesor investigador en la Universidad Autónoma Metropolitana-Cuajimalpa (UAM-C) en México, División de Ciencias de la Comunicación y Diseño. Departamento de Tecnologías de la Información. Es integrante de la Red de Fortalecimiento de la Docencia de la UAM-C. Es miembro del Sistema Nacional de Investigadores del Consejo Nacional de Ciencia y Tecnología (CONACYT) y del Consejo Mexicano de Investigación Educativa (COMIE). Ha sido profesor-investigador de la Maestría y el Doctorado en Ciencias de la Educación en la Universidad Autónoma del Estado de Hidalgo (UAEH), donde fue coordinador académico del Doctorado en Ciencias de la Educación y jefe del Área Académica de Ciencias de la Educación. Ha sido coordinador de las líneas de investigación Currículum, Innovación Pedagógica y Formación; y Diagnóstico, Evaluación y Planeación de la Educación en el CINCIDE. Posee 56 artículos de investigación publicados en revistas nacionales e internacionales y 24 capítulos de libro. Es autor de los libros: La evaluación de alumnos en la universidad: un estudio etnográfico (2010) y Evaluación del aprendizaje y para el aprendizaje. Reinventar la evaluación en el aula (2016).
} 
doi: http://dx.doi.org/10.15359/ree.21-2.6

URL: http://www.una.ac.cr/educare

CORREO: educare@una.cr

\begin{abstract}
Teaching collegiality in the Mexican primary school is influenced by a global dynamic that forms a complex school culture, with singular educational practices that have been established over time. A qualitative investigation of ethnographic character was conducted by using the case study modality. The case consisted of 13 professionals in charge of the primary education management. The techniques used to collect data were participant observation, semi-structured interview and analysis of documents. Through an inductive research process, empirical categories of analysis were constructed to finally produce an interpretative text that identified some practices of teacher collaboration; these practices are described and analyzed in this article. Among the main findings, it was identified that what motivates the teaching staff to collaborate with others has to do with resolving practical and immediate situations with preciseness; so, the school can continue to function with "a sense of normality». It was also confirmed that the time for teaching collegiality is not scheduled; then, the teachers often consume their students' recess time, and even the management staff extends that time to develop different meetings related to providing several types of information.
\end{abstract}

Keywords: Teacher collaboration; school management; school culture; educational practices; primary education.

\title{
Introducción
}

Este artículo se deriva de la tesis doctoral titulada: El papel del director en el contexto educativo: La evaluación y la colegialidad como exigencias de la escuela primaria. El caso de la zona escolar N. 127 (Martínez-Cruz, 2013). Se trató de una investigación etnográfica mediante la modalidad de estudio de casos, cuyo objetivo general consistió en recoger evidencias acerca del papel del personal a cargo de la dirección de escuela primaria ante las exigencias de implementar procesos de evaluación y colegialidad docente, como mecanismos para mejorar la calidad de la educación.

Este artículo aborda la categoría empírica denominada "La colegialidad docente en la escuela primaria mexicana: Algunas prácticas que la representan". Se trató de caracterizar la cultura de la colegialidad docente en la escuela primaria y de identificar el papel directivo en ese entramado, para lograr una mejor comprensión de las prácticas de colaboración y sus diversas implicaciones en el contexto educativo mexicano. En la primera parte se incluyen algunas referencias teóricas en torno al tema. En la segunda se describe la metodología que orientó la investigación. En la tercera se analizan las evidencias empíricas obtenidas, mediante el proceso investigativo efectuado en las diferentes escuelas primarias, en torno a ciertas concepciones y prácticas de colegialidad docente. La cuarta parte es una conclusión acerca de la temática abordada.

El siglo XXI se caracteriza por la globalización de la economía y de la educación. Los medios electrónicos han intensificado la comunicación entre sujetos de diferentes latitudes y puntos cardinales del planeta. El comercio se ha intensificado y la educación básica parece haberse unificado en torno a un perfil de egreso universal que cada país pretende alcanzar, 
independientemente de su contexto, condiciones y posibilidades. La evaluación es el medio más importante para valorar este proceso y la colegialidad el espacio de diálogo para encontrar alternativas colectivas a los desafíos que plantea el momento que se vive.

En un mundo complejo, desafiante y cambiante, la educación evoluciona a cada instante, lo que demanda, como cita Stoll, Fink y Earl (2004), que los sujetos aprendan, desaprendan y reaprendan ante una dinámica económica, social y cultural que a diario se renueva. Este nuevo escenario en el ámbito de la escuela primaria conforma una cultura escolar propia, caracterizada por una constante evaluación y por esfuerzos de colegialidad docente, cuyo origen está en la necesidad del profesorado de dialogar acerca de los problemas comunes que viven cotidianamente en la escuela. A través de un estudio de caso de corte etnográfico, se realizaron observaciones y entrevistas, cuyos datos se analizaron e interpretaron a la luz de planteamientos teóricos, empíricos y culturales. Para finalmente presentar algunos resultados que tratan de responder a las cuestiones planteadas inicialmente: ¿cuáles son las razones que impulsa al profesorado a colegiar?, ¿quiénes participan del trabajo colegiado en la escuela primaria?, ¿cuándo se reúne el profesorado para colegiar? y ¿cómo se desarrolla la colegialidad docente en la escuela primaria?

Concluimos señalando que la colegialidad docente se desarrolla principalmente de tres formas. Primera, como cita Hargreaves (2005), se trata de una colaboración artificial, donde por lo general el personal directivo utiliza el espacio de las reuniones para dar al profesorado indicaciones, disposiciones y lineamientos a seguir en el trabajo de la escuela. Segunda, el profesorado se reúne cuando emergen diversas problemáticas "urgentes" que a menudo requieren ser atendidas o solucionadas en un corto tiempo. Tercera, la colegialidad surge ante la necesidad del profesorado por conocer acerca de los nuevos requerimientos que le demanda la reforma educativa actualmente en curso.

\section{Referentes teóricos}

\section{Globalización y cultura escolar}

Vivimos en un mundo globalizado donde las sociedades humanas del siglo XXI se encuentran inmersas en la complejidad, la incertidumbre y los grandes desafíos. En efecto, los actores nacionales comparten escenarios, conocimiento e información con distintas organizaciones e instituciones educativas, con movimientos sociales y políticas internacionales, así como con empresas trasnacionales. Ante ello, García (2005) plantea como disyuntiva globalizarnos o defender nuestra identidad, aunque no se trata de tomar una vía u otra, sino de encontrar las mejores posibilidades. 
doi: http://dx.doi.org/10.15359/ree.21-2.6

URL: http://www.una.ac.cr/educare

CORREO: educare@una.cr

La globalización ha influido y orientado la educación a través de las políticas educativas elaboradas por organismos internacionales y difundidas a los países a través de mecanismos como la Conferencia mundial sobre educación para todos, celebrada en Jomtien, Tailandia (WCEFA, 1990), que plantea garantizar el acceso universal para satisfacer las necesidades básicas de aprendizaje de cada persona. El Foro Mundial sobre Educación, celebrado por la Unesco (2000), reafirmó el compromiso de asegurar el acceso a la educación primaria de alta calidad. En tanto que la Cumbre del Milenio (ONU, 2000) propuso lograr la enseñanza primaria universal, promover la igualdad entre géneros y la autonomía de la mujer.

Estas políticas se han implementado (en algunos aspectos) en México a través de la Secretaría de Educación Pública en educación básica, apoyándose en el personal directivo para su aplicación; al que se le demanda un conjunto de competencias profesionales entre las que destacan el liderazgo educativo, la habilidad para orientar la acción de los grupos, la capacidad para facilitar el crecimiento personal y profesional de sus docentes; así como habilidades para motivar, desarrollar y consolidar al equipo de trabajo e integrar a los padres y madres de familia en la formación de sus hijos e hijas.

Por otro lado, diversos especialistas en educación (Castoriadis, 1997; Gimeno, 2002; Habermas, 2006; Pérez, 2004; Morin, 1999) refieren que la cultura está constituida por un conjunto de significados, valores, saberes, creencias, formas de sentir y expresarse que se comparten e intercambian en un grupo determinado como la escuela, el centro de trabajo o la familia, entre otros.

Inserto en una cultura escolar singular y compleja, el personal directivo construye una identidad profesional propia que lo distingue en relación con labores desempeñadas en otras direcciones de escuela, en ocasiones, su pertenencia a esta cultura conlleva prestigio y, en otras, descrédito. Según Hargreaves (2005), la cultura escolar está enmarcada por su contenido y forma: el contenido emerge del interior del sujeto a través de creencias, mitos, valores, hábitos, normas, reglas e ideas que encuentran forma en acciones que se desarrollan y manifiestan a través de símbolos y lenguaje para comunicar situaciones precisas y visibles ante las demás personas.

En síntesis, la cultura escolar se presenta como propia y única, paralela a la globalización, el neoliberalismo y la postmodernidad, o tal vez, producto de ellas. En 1995, Dominique Juliá empezó a utilizar el término cultura escolar para caracterizar las diversas acciones desarrolladas y emprendidas por actores que de manera directa e indirecta intervienen en el espacio denominado escuela. Lo que significa que su conformación está integrada por creencias, valores, conocimientos, mitos y significados, manifiestos en acciones educativas de los diferentes actores de la comunidad escolar, dentro de un espacio y tiempo determinados. Por la importancia que tiene la cultura escolar para comprender el ethos de los centros educativos, en este artículo analizaremos algunas de sus implicaciones en torno al acontecer cotidiano de las comunidades implicadas mientras conviven, se relacionan, interaccionan y aprenden. 


\section{La colegialidad docente en distintos niveles educativos}

La colegialidad es un concepto polisémico, complejo y en ocasiones controversial, que tiene connotaciones distintas, según la procedencia e intención de quien lo emplea. Lo habitual es relacionarlo con colegas de un campo común, profesión, centro de trabajo, campo del conocimiento, espacio geográfico, entre otros. En educación básica, en especial en el nivel de primaria, se expresa formalmente a través del Consejo Técnico Consultivo; según el Acuerdo 96 (SEP, 1982), dicho Consejo debe sesionar por lo menos una vez al mes, para analizar diversos temas, tales como: práctica docente, planes y programas de estudio, métodos de enseñanza, evaluación de programas; así como la adquisición, elaboración y uso de materiales didácticos e impulsar la capacitación docente.

Por su parte, en el Acuerdo Nacional para la Modernización de la Educación Básica, el Consejo Técnico Consultivo, se asume como "núcleo de la actualización... [que] involucrará a los ... directores" (SEP, 1992, p. 13). Posteriormente, el Programa Nacional de Educación 2001-2006 (SEP, 2001), incorpora la gestión escolar y la "participación corresponsable de alumnos, docentes, directivos y padres de familia" (p. 139). Después, el Programa Sectorial de Educación 2007-2012 (SEP, 2007) incluye un nuevo modelo de gestión escolar institucional que convoca a la participación de los centros en la toma de decisiones, la corresponsabilidad de los actores educativos en los Consejos Escolares de Participación Social, a través de modelos de gestión estratégica y modelos de gestión participativa amplios y flexibles. Actualmente, el Programa sectorial de educación 2013-2018 (SEP, 2013b) sustituye el Consejo Técnico Consultivo por el Consejo Técnico Escolar, asignándole espacio, tiempo y temas a tratar cada mes, con el objetivo de fortalecer el desarrollo profesional del personal directivo y docente para mejorar la calidad educativa.

Para el caso de la educación media superior, la Dirección General de Bachillerato (2007) considera al trabajo colegiado como una estrategia dirigida al personal docente y directivo "para que identifiquen sus principales problemas educativos, propongan estrategias de solución, mediante el diseño y puesta en marcha de un proyecto escolar" (p. 8). Por su parte, el subsistema de Educación Normal (SEP, s. f., p. 2) plantea el desarrollo del trabajo colegiado como un "medio fundamental para conformar un equipo capaz de dialogar, concertar, compartir conocimientos y experiencias en torno a asuntos y metas de interés común". Asimismo, De Jesús, De la Rosa y García (2003) proponen el trabajo colegiado para la formación permanente de docentes y como una estrategia para que una comunidad diversa se reúna a compartir, debatir, reflexionar y construir un proyecto de docencia, de profesión y de universidad. A su vez, Lugo (2004) plantea la construcción de espacios colegiados que relacionen a quienes investigan, toman decisiones y aplican el currículo, para que enfoquen los esfuerzos hacia la actualización y mejoramiento de los contenidos, y la retroalimentación sobre las metodologías de enseñanza y aprendizaje. 
doi: http://dx.doi.org/10.15359/ree.21-2.6

URL: http://www.una.ac.cr/educare

CORREO: educare@una.cr

En el ámbito universitario, Pujadas y Durand (2002) afirman que se alimenta la cultura del egoísmo, el individualismoyla fragmentación, la cual hay quecambiar por una cultura de participación y construcción de una comunidad comprometida con el diálogo que rompa con el aislamiento. Mientras que otros estudiosos como Lobato y De la Garza (2009) refieren que la colegialidad es la búsqueda de un proyecto académico común, establecido a través de un proceso integrativo de los cuerpos académicos, a partir de las demandas del entorno, para la toma de decisiones individuales y grupales que dependen del acervo de premisas comunes del profesorado.

En consecuencia, la colegialidad docente se emplea como una estrategia o un medio que permite a un grupo de personas interactuar con propósitos comunes, intercambiando ideas de manera formal e informal, en un espacio físico y simbólico como la escuela, para mejorar e incluso ser más productivo. La colegialidad entre el profesorado también representa un espacio de encuentro que permite aprender, convivir y construir una cultura de trabajo en equipo. Para algunos especialistas, la colegialidad o colaboración docente es fuente y objeto de investigación, así como de publicaciones con planteamientos diversos y nuevas formas de entendimiento.

Sánchez, Nishikawa, Cordero y Bocanegra (2008) plantean que existe una colegialidad invisible en la escuela primaria cuyo germen es el aprendizaje, que es desarrollado de manera natural y espontánea en paralelo con el individualismo y el aislamiento. Por su parte, Moreno (2006) refiere que la política educativa ve la colaboración y la colegialidad docente como una estrategia para conseguir mejores resultados en los centros educativos, pero existe la sospecha de ser una opción para cooptar a los maestros y maestras, y responder a intereses ajenos a los del colectivo docente. Ante ello, es importante el compromiso asumido entre la persona a cargo de la dirección y el profesorado para colaborar de forma productiva con todos los agentes escolares, para afrontar las dinámicas de una sociedad globalizada y compleja como la actual, con nuevos argumentos, ideas y actitudes.

En este tenor Fullan y Hargreaves (1999) enfatizan que el trabajo en equipo es fundamental para el cambio educativo, pero reconocen que este enfrenta dificultades en su instrumentación, las cuales pueden ser revertidas por el personal directivo y docente a través de su liderazgo, disposición y altas expectativas para consolidarlo. La colaboración debe ser vista como un proceso a largo plazo, donde se creen oportunidades para que los cuerpos docentes trabajen juntos, promuevan y faciliten la asociación entre sí.

Asimismo, Hargreaves (2005) identifica cuatro formas de cultura del profesorado: individualismo, colaboración, colegialidad artificial y balcanización. El individualismo es caracterizado por aislar el trabajo docente, cerrar los canales de comunicación, por lo que constituye un obstáculo para la labor de equipo. La cultura de colaboración favorece las relaciones de trabajo en equipo que se manifiestan como espontáneas, voluntarias y orientadas al desarrollo. En tanto que la colegialidad artificial está reglamentada y obliga a su 
implementación en un tiempo y un espacio en el centro de trabajo. Finalmente, la balcanización es consecuencia de la interrelación entre docentes que construyen pequeños subgrupos en la escuela, con objetivos propios, en ocasiones alejados del objetivo común.

Ahora bien, Fullan (2002) plantea que a través de la colegialidad docente se desarrollan comunidades profesionales de aprendizaje, donde aprenden a administrar su tiempo, a mejorar de manera colectiva, a utilizar su poder para implementar el cambio, a conceptualizar el trabajo como un continuo de aprendizaje, caracterizado por las habilidades docentes, el ambiente de clase y las características profesionales; lo cual marca una diferencia con las escuelas mal estructuradas, donde el profesorado está poco comprometido con su labor. En resumen, pensamos, entonces, que la colegialidad docente es un espacio de diálogo y reflexión entre colegas, en torno a un proyecto común con metas compartidas. Esta acción comunicativa -como la llama Habermas (2006) - tiene como punto de partida el interés de los sujetos implicados por conocer, investigar y diseñar alternativas de solución a situaciones problemáticas o a necesidades existentes. Lo anterior, sin excluir la posibilidad de que cada maestro o maestra busque, de manera autónoma, la satisfacción de las necesidades que experimenta en su contexto. Como afirma Heller (1996): "Nadie sino el portador de necesidades está autorizado a seleccionar entre ellas y a distinguir entre las verdaderas y las falsas, las reales y las irreales" ( $p$. 108), lo que según Wenger (2011) convierte a los maestros en una comunidad de aprendizaje que tiene un compromiso mutuo, una empresa y un repertorio compartidos.

\section{Metodología de investigación}

Se trató de una investigación cualitativa de corte etnográfico mediante la modalidad de estudio de casos. El caso lo constituyeron 13 directores y directoras de educación primaria que se desempeñan en una zona escolar ubicada en la región del Valle del Mezquital, en el Estado de Hidalgo (México). La zona escolar donde se llevó a cabo la investigación está integrada por 13 escuelas (12 de turno matutino y una de turno vespertino). En conjunto, estas escuelas atienden a 1633 estudiantes en 12 comunidades rurales y agrícolas; en la cual laboran 92 docentes unigrado, un docente multigrado. En cuanto al personal directivo, 3 atienden grupo y 10 con la categoría de directores técnicos, además del personal administrativo y de intendencia.

Algunas características importantes del personal directivo son los años que tienen de servicio dentro del sistema educativo. Se encontró que de los 13 sujetos directores investigados, 8 tienen más de 30 años de servicio, 4 entre 25 y 29 años y 1 tiene 18 años. Su experiencia profesional en la función directiva se distribuye de la siguiente forma: 3 tienen entre 20-29 años, 2 entre 15-17 años, 4 entre 6-10 años y 4 tienen 1-5 años. En cuanto a su situación contractual se encontró que 10 cuentan con una clave que corresponde a su función directiva, llamados técnicos porque no tienen grupo, cuya función consiste en dirigir y coordinar la escuela; 3 son docentes que atienden a un grupo-clase, además de realizar funciones directivas. 
doi: http://dx.doi.org/10.15359/ree.21-2.6

URL: http://www.una.ac.cr/educare

CORREO: educare@una.cr

La elección de los sujetos directivos que conformaron la población objeto de estudio respondió a intereses y necesidades de los investigadores, teniendo como referencia la pregunta central de investigación: ¿cómo desarrolla su papel el director o directora de educación primaria, en un contexto educativo que exige evaluar y colegiar?; así como el objetivo general de la investigación que consistió en recoger evidencias acerca de su papel en la escuela primaria ante las exigencias de implementar procesos de evaluación y colegialidad para mejorar la calidad de la educación. Para la recolección de la información se utilizaron las siguientes técnicas: observación participante, entrevista semiestructurada y análisis de documentos.

El proceso investigativo se desarrolló en distintas etapas. En un primer momento, se observaron y registraron de manera minuciosa las actividades del personal directivo durante cinco días diferentes de la semana en un lapso de seis meses, lo que constituyó un total de 65 registros de notas de campo. En un segundo momento, se transcribieron los registros de observación y se procedió a subrayar los fragmentos en los textos para posteriormente preguntar, inferir e identificar los patrones emergentes que dieron origen a los grandes temas, agrupados en torno a los objetivos particulares. De la información anterior, surgió la construcción del guion de la entrevista semiestructurada que se realizó a cada sujeto director para profundizar en su conocimiento sobre la temática investigada. En un tercer momento, algunos de los grandes temas previamente identificados dieron lugar a la construcción de las categorías de análisis que abarcaron el mayor número de patrones emergentes. En un cuarto momento, se triangularon los datos obtenidos a partir de contar con un cuerpo de categorías empíricas -categorías sociales-, organizadas de acuerdo con un cuerpo categorial propio-categorías de los intérpretes-, lo que propició una comunicación paralela con los hallazgos y conceptos producidos por los autores -categorías teóricas-. Finalmente, se arribó a la producción de un texto interpretativo en el que se emplearon claves para identificar a los directivos y directivas (preservando así su anonimato), dicho texto contiene información relevante obtenida a través de establecer relaciones y encontrar significado a las acciones cotidianas del personal directivo.

\section{Análisis de resultados}

En esta investigación se reconoce que la colegialidad docente implica interactuar y relacionarse en torno a propósitos comunes, intercambiando perspectivas e ideas, sea de manera formal e informal, en un espacio físico y simbólico como la escuela. No obstante, en ocasiones, la colegialidad docente también puede ser empleada como una estrategia para controlar, jerarquizar y cooptar al profesorado; o bien para mejorar, relacionarse y lograr más producción; lo que favorece la reflexión y el diálogo entre colegas. Sin duda, la colaboración docente bien empleda puede representar un medio valioso para el desarrollo profesional, la implementación del cambio educativo y el intercambio de experiencias para mejorar la educación. 
En este sentido, la colegialidad docente se desarrolla a partir de principios básicos como la toma de decisiones, el trabajo colaborativo, la interacción, el análisis, el aprendizaje y la práctica reflexiva; por consiguiente, se forma y renueva un equipo a través de asambleas democráticas, caracterizadas por la tolerancia y el diálogo entre docentes a partir de situaciones comunes.

Implementar la colegialidad docente en educación básica ha planteado serios desafíos, que van desde su concepción hasta su operación, de ahí el interés por analizar las respuestas de las siguientes cuestiones: ¿cuáles son las razones que impulsa al profesorado a colegiar?, ¿quiénes participan del trabajo colegiado en la escuela primaria?, ¿cuándo se reúne el profesorado para colegiar? y, ¿cómo se desarrolla la colegialidad docente en la escuela primaria?

Porque más allá del llamado de la autoridad oficial a trabajar de manera colegiada, las reuniones de profesores y profesoras existen ante problemáticas y situaciones comunes de trabajo, las cuales aparecen en un"amplio intervalo donde se sitúan diferentes comportamientos que van desde la cultura de colaboración espontánea a la colegialidad burocrática y artificial" (Pérez, 2004, p. 170).

\section{Propósitos del trabajo colegiado}

En escuela primaria mexicana, cotidianamente, los actores educativos se reúnen con diferentes propósitos, entre ellos, resolver problemas, atender necesidades de información y conocimiento, informar sobre disposiciones oficiales, evaluar el desempeño docente, planear y organizar diversas tareas, proporcionar información sindical y, prepararse para los exámenes estandarizados. Todo ello (y más) conforma el ambiente natural y rutinario de la vida escolar que describiremos, analizaremos e intentaremos comprender, a partir de la información procedente de las entrevistas y los registros de observación.

Cada quien de los 13 directores y directoras de educación primaria que formaron parte de este estudio tiene su propia noción de lo que significa la colegialidad. Sus respuestas permiten agrupar la información en dos bloques: primero, 7 consideraron que la colegialidad consiste en trabajar en equipo a partir de un fin común, un tema, un proyecto o una problemática; donde se aportan distintos puntos de vista y se establecen acuerdos. Segundo, 6 manifiestaron que la colegialidad es un espacio de organización, integración, colaboración y construcción de soluciones, a partir de necesidades y dificultades que se presentan en la escuela. Como evidencia, un director reconoce que la colegialidad:

EMMC: Es un espacio donde todos participamos para conocer algún tema, proyecto y problemática que hay en la escuela. Asimismo, nos involucramos para resolverlo (E.EMMC.280812). 
doi: http://dx.doi.org/10.15359/ree.21-2.6

URL: http://www.una.ac.cr/educare

CORREO: educare@una.cr

Al respecto, Stoll et al. (2004) afirman que "se trata a la colegialidad como un rasgo central del aprendizaje y desarrollo del docente, que enfatiza la ayuda mutua y el compartir" (p. 110). Sin embargo, Mitchell y Sackney (2000, citados por Stoll et al., 2004), plantean que la colegialidad se conforma en una comunidad de aprendizaje a partir de que los involucrados "adoptan un enfoque activo, reflexivo, de colaboración, orientador del aprendizaje y promotor del crecimiento, hacia los misterios, problemas y perplejidades de la docencia y de la enseñanza" (p. 148), lo que, sin duda, implica dialogar, investigar y aprender.

En cuanto a la finalidad del trabajo colegiado, 3 refirieron a la mejora del funcionamiento de la escuela, la práctica docente y el trabajo del director o directora; 6 indicaron que el propósito del trabajo colegiado consiste en resolver las problemáticas pedagógicas, sociales, culturales, de aprendizaje y rezago educativo. Otros 2 directores afirmaron que dicho propósito consiste en planear actividades, llevarlas a cabo y evaluarlas; mientras que 2 más opinaron que el personal docente se reúne para actualizarse, organizarse y evaluar las acciones. Al respecto, un director declara que:

ACA: El propósito fundamental es hablar de lo mismo todos y conocer la problemática de cada grado. ... solventar los problemas pedagógicos ... problemas sociales, conflictos, etc. (E.ACA.100912).

En este sentido, Jaworski (1998, citado en Stoll et al., 2004, p. 158), afirma que a las personas las anima a participar un conjunto de significados compartidos en acciones conjuntas donde hablan, piensan, descubren y alcanzan objetivos comunes. Por su parte, Wenger (2011) se refiere a la constitución de comunidades de práctica donde los sujetos que interactúan tienen compromisos comunes, participan, se identifican y establecen vínculos de competencia comunitaria, lo que conforma un lugar privilegiado para el aprendizaje y la adquisición de conocimientos, en este caso, desde la escuela primaria.

\section{Beneficios del trabajo colegiado}

A 8 directores y directoras, la colegialidad les permitió consolidar un equipo de trabajo caracterizado por la confianza, orientación, apoyo, convivencia, mejores relaciones y organización. Mientras que 5 afirmaron que la colegialidad ha contribuido a la mejora de la práctica docente, las estrategias de trabajo del profesorado y los resultados de la prueba Enlace. Al respecto, tenemos el siguiente registro:

HMB: ... permite organizar el trabajo, se convive ... se mejoran las relaciones entre personas y entre los maestros (E.HMB.220812). 
Lo anterior parece poner en evidencia el tránsito lento y gradual de una cultura aislada e individualista a una cultura de colegialidad. Al respecto, Hargreaves, Earl y Ryan (2008) afirman que "las culturas de la colaboración convierten el aprendizaje individual en aprendizaje compartido" (p. 280), poniendo énfasis en la estructura, relaciones y diseño de tareas que incrementen las oportunidades de aprender, por lo que se comparte y afronta en el trabajo profesional.

Por otra parte, los datos obtenidos mediante las observaciones realizadas en esta investigación confirmaron la información procedente de las entrevistas. Pero, además, permitieron identificar y caracterizar reuniones administrativas en diferentes momentos de la jornada laboral para tratar asuntos relacionados con la entrega y revisión de documentos, que regularmente son requeridos de un día para otro; lo que ocasiona malestar en algunos profesores y profesoras, alegría en otros, porque abandonan su grupo clase. También se organizan reuniones para dar al profesorado información sobre indicaciones y disposiciones oficiales, producto de una dirección vertical, que impone disciplina y control a través de diversas sanciones administrativas.

En tanto, la sobrecarga administrativa, el cumplimiento de responsabilidades normativas y el control a través de diversas indicaciones y disposiciones van conformando obstáculos que minan el desarrollo de verdaderos espacios de diálogo y aprendizaje al interior de la escuela primaria. Ante lo cual, Fullan y Hargreaves (1999) afirman que "una nueva escuela requiere que los directores conozcan a fondo la misión de la escuela, sus implicaciones para cada grado escolar, para el trabajo de cada maestro y para la organización escolar en su conjunto" (p. 9). Y que además no pierdan de vista la importancia del trabajo colegiado como parte sustancial para mejorar.

El clima de las reuniones de evaluación de docentes varía de una escuela a otra, en parte, influido por la actuación del director o directora, quien imprime a estas una atmósfera particular, según su estilo directivo. Así, se identificó a 4 que aplican los lineamientos normativos de evaluación en cuanto a la asignación del puntaje para el programa de estímulos, lo que muchas veces genera confrontación y división entre docentes; en tanto que 9 conceden al profesorado plena libertad para autoasignarse los puntos en su evaluación, lo que causa beneplácito entre docentes y genera un ambiente de "todas las personas contentas y en paz". La evaluación docente se reduce a alcanzar un estímulo económico; olvidándose del aprendizaje, la retroalimentación, los procesos de mejora, la evaluación formativa y la calidad educativa. Además, la evaluación desde esta lógica parece alejada de los objetivos deseados para mejorar el desempeño docente y directivo, lo cual, según Hargreaves et al. (2008), no resulta adecuado para mejorar la calidad de la educación, lo que obliga a revisar y confrontar las culturas más arraigadas de la escuela. 
doi: http://dx.doi.org/10.15359/ree.21-2.6

URL: http://www.una.ac.cr/educare

CORREO: educare@una.cr

En esta investigación también identificamos que el personal directivo y docente se reúne al inicio del año escolar para organizar y planear la asignación de los grupos de clase a cada persona; después para formarse y prepararse, así como para el desarrollo de actividades sociales. En este sentido, la asignación del grupo-clase en ocasiones genera malestar, confrontación y división entre el profesorado. La preparación y formación continua del profesorado se enfoca, principalmente, en la mejora de los resultados de la prueba Enlace; en tanto se identificó que las actividades sociales consumen mucho tiempo del proceso de enseñanza aprendizaje, lo que obstaculiza el desarrollo del trabajo y dificulta alcanzar las metas planteadas en el plan y programas de estudio.

En general, las reuniones del personal docente se podrín caracterizar como artificiales, porque aunque estaban orientadas al trabajo conjunto en diversas actividades, buscaban controlar el riesgo, la aventura y la incertidumbre en los procesos naturales de colaboración espontánea. Como afirma Pérez (2004), las reuniones deberían "considerarse un paso previo y muchas veces imprescindible para provocar la deseada transición hacia la colaboración" (p. 171) y la oportunidad de consolidar un equipo de aprendizaje con objetivos comunes y compromisos para cambiar.

Por otro lado, se identificaron reuniones sindicales en las que con anuencia del personal directivo se informa al profesorado sobre asuntos de trabajo, tales como el salario, conquistas laborales y actividades sindicales. Además, en dichas asambleas se favorece la manifestación de inquietudes y pronunciamientos a favor o en contra de la organización sindical, llegando, incluso en algunos casos, a la confrontación. Igualmente, se tuvo el registro de reuniones en las que se abordaron diversas problemáticas que tanto el personal directivo como docente afrontan diariamente en la escuela primaria, situaciones que les obligan a desafíos cada vez más complejos que requieren del diálogo y la reflexión para el diseño de alternativas de solución.

Ante el panorama descrito, concluimos que habitualmente el personal directivo y sus docentes se reúnen para llevar a cabo actividades operativas e instrumentales necesarias para el funcionamiento inmediato de la escuela; además, ante dificultades y necesidades a resolver en un corto plazo, para mantener el funcionamiento regular de la escuela, lo que genera un conservadurismo que, según Pérez y Gimeno (1993, citados en Pérez, 2004, p. 179) "impone sobre los individuos, que viven en ella ... una manera de pensar, sentir y actuar, especialmente sobre la educación y la escuela, fuertemente arraigada, que perdura en el tiempo y ahoga, tanto los intentos individuales de innovación, como las posibilidades de crítica teórica".

En consecuencia, el personal directivo y docente trabaja con propósitos a corto plazo para afrontar lo inmediato, sin cuestionar las prácticas cotidianas; lo que, según Moreno (2006), no es un objetivo deseable en un grupo que comparte una forma de cultura y filosofía que ha surgido de las creencias personales durante el proceso educativo. Es necesario poner en duda este estado de 
cosas para romper con la inercia y construir comunidades dispuestas a vivir en la incertidumbre, el cambio y en aprendizaje que, según Wenger (2011), sean producto de negociar nuevos significados, de la experiencia social, de la participación, del compromiso y de la imaginación.

\section{Agentes participantes de la colegialidad}

Las reuniones en la escuela primaria tienen diferentes escenarios y actores. Sin embargo, el protagonista principal es el ente director, quien, entre sus acciones recurrentes informa, orienta, asesora, sensibiliza, concientiza, invita, negocia, destensa, ordena y controla, por citar algunas; tales actividades las describiremos en esta sección para intentar comprender la participación de los diferentes actores en las reuniones que se convocan o surgen en el contexto de la escuela primaria.

De inicio, identificamos al personal directivo que informa al profesorado de asuntos relacionados con la educación, la escuela y el trabajo. Asimismo, instruye sobre disposiciones que el personal docente debe cumplir con respecto a sus obligaciones, como parte de la normatividad laboral. Con ello se favorece una forma de dirigir basada en el control, la jerarquía, la obediencia y la subordinación. También encontramos que el personal directivo y el docente invierten parte de su tiempo laboral en organizar actividades sociales, para lo cual se asignan comisiones y se reparten responsabilidades. Algunos profesores y profesoras realizan estas tareas con responsabilidad y profesionalismo, otros, con desinterés y apatía.

Asimismo, descubrimos que estos actores educativos afrontan diferentes problemáticas en su desempeño profesional, lo que hace necesario el diálogo para intercambiar opiniones y sugerencias, construir acuerdos y alternativas de solución; esto es lo que, según Ball y Cohen (1999, citados en Stoll et al., 2004), permite la construcción de un espacio de aprendizaje profesional continuo, donde se aprende de la práctica, en tanto que Wenger (2011) considera que los sujetos implicados aprenden de manera conjunta al negociar el significado de forma creativa, desde su práctica profesional.

Además, también se pudo constatar la participación del personal directivo y el docente en los programas de formación continua organizados e implementados por la autoridad educativa; acciones que, como señalan King y Newmann (2001, citados por Stoll et al., 2004, p. 165), serán "efectivas para estimular la calidad de la enseñanza y el aprendizaje si se centran en la comunidad profesional junto con todo el personal, además de en el conocimiento, las habilidades y las disposiciones de los docentes".

En ciertos momentos del ciclo escolar el personal directivo convoca a sus docentes al proceso de evaluación de carrera magisterial, siguiendo un protocolo establecido. Se observó que en algunos casos el personal directivo flexibilizaba la evaluación ante la presión de docentes, generando un proceso viciado y de simulación. Por otro lado, encontramos que el 
doi: http://dx.doi.org/10.15359/ree.21-2.6

URL: http://www.una.ac.cr/educare

CORREO: educare@una.cr

ente director y los padres y madres de familia abordaban de forma conjunta problemas como la educación y evaluación de los niños y las niñas, la conformación y constitución del comité de la Asociación de Padres de Familia y el comité de Participación Social; pero parece que el interés de la escuela por hacer partícipe a la familia responde al hecho de garantizar su apoyo económico, humano y material. En otro momento, identificamos la anuencia del personal directivo para efectuar reuniones sindicales donde se informa de logros laborales, problemas del sindicato con el patrón, vacantes en diferentes espacios educativos y demás problemáticas laborales. En ocasiones, en estas reuniones se elige a quien representa a la escuela, al Comité Delegacional, al personal delegado, al congreso político y al congreso de educación o ambos. Como muestra de ello, está la siguiente evidencia.

EMC. 10:30. [Recreo] Los maestros compran alimentos en la tienda escolar y se reúnen en un salón, el representante sindical de la escuela (JLAL) informa la situación que vive la zona escolar y el sindicato estatal ...; mientras afuera unos alumnos pelean y rompen un vidrio.

Alumnos: [A coro] ¡Maestros, rompieron un vidrio!

Maestros: [A coro] ¡Estamos en reunión, por favor, luego los atendemos! (O.EIRD.290412).

Ante la falta de espacio y tiempo para las reuniones entre el personal directivo y los maestros y maestras, los grupos docentes utilizan el recreo para dialogar, informar y acordar, mientras el alumnado juega, come e incluso en ocasiones sobrepasa los límites de la convivencia.

En conclusión, la participación de los actores educativos (personal directivo, docentes y estudiantes) en diferentes espacios y escenarios favorece la comunicación, colaboración, acuerdos y desacuerdos, identificación y negociación de significados, aprendizaje y conocimiento desde la práctica, pues, como cita Wenger (2011), "el aprendizaje de una organización reside en las oportunidades de negociar significados de una manera creativa" (p. 309).

Por otro lado, la participación, sobre todo del director o directora y personal docente, tiene como punto de partida situaciones formales y normativas del trabajo, lo que a menudo favorece relaciones frontales de autoridad y control. Por el contrario, es evidente que estos actores también se reúnen ante las necesidades de conocimiento y situaciones que implican resolver problemas de la práctica profesional, lo que genera un espacio de diálogo e interacción en el que se investiga, comparte, confronta y aprende entre sí, conformando una colegialidad docente que apuesta por el aprendizaje colectivo. 


\section{Desarrollo del proceso de colegialidad}

Las reuniones escolares entre el personal directivo y el docente están inmersas en un marco cultural que, según Hargreaves (2005), determina su contenido y forma. Entendiendo al contenido como las creencias, valores y hábitos compartidos por un grupo, y a la forma como las acciones, desarrollo y manifestación del contenido. En espacios como el Consejo Técnico Consultivo, los talleres de actualización y formación continua, las reuniones de evaluación, las reuniones informativas para tratar disposiciones generales y asuntos emergentes, surge una dinámica de colaboración en la que se establecen acuerdos en temas comunes de interés para los actores educativos.

El personal directivo coincide en señalar que las reuniones formales e informales se generan a partir de las necesidades de información, del surgimiento de actividades cívicas y sociales, lo que favorece la participación y responsabilidad en la integración del equipo de trabajo. No obstante, también hay quien manifiesta que existen obstáculos como la falta de espacio y de tiempo, la impuntualidad, el desinterés y las diferencias entre docentes, lo que confirma que "son pocos los espacios y los tiempos de que dispone el profesorado para la colaboración" (Moreno, 2006, p. 117). Por esto se hace necesario favorecer la confianza, brindar apoyo y promover mejores relaciones entre los actores educativos, para consolidar un verdadero equipo de trabajo con capacidad para la innovación y el cambio.

Por su parte, la autoridad educativa promueve la colegialidad docente en la escuela primaria; pero, como precisa Smith (1999, citado por Moreno, 2006, p. 107),"se trata del síndrome de dar libertad mientras se está amordazado" que en nada favorece el trabajo colegiado. Por otro, busca controlar a través del ente directivo, suministrando información para la planeación y ejecución de diversas acciones, lo que genera más que un proceso natural de diálogo, un proceso de control a través de la obediencia y sumisión.

Tenemos, entonces, que las necesidades de información y de conocimiento movilizan al personal directivo y a parte del personal docente a indagar, dialogar y compartir, a pesar de la apatía y desinterés de algunos miembros, y la falta de espacio y tiempo para su desarrollo. Se promueve la reflexión y negociación de significados entre el profesorado. Como señala Wenger (2011): "en la práctica, nos centramos en los significados que podemos negociar por medio del aprendizaje y esos significados constituyen la fuente de la energía necesaria para aprender" (p. 314).

Por otro lado, las reuniones frontales evidencian el ejercicio del poder directivo a través de prácticas arraigadas que por años han funcionado en las escuelas primarias como formas de control y manejo cómodo de la institución educativa. Así lo revela la siguiente nota de observación. 
doi: http://dx.doi.org/10.15359/ree.21-2.6

URL: http://www.una.ac.cr/educare

CORREO: educare@una.cr

11:50 Al salón del director de la escuela acuden alumnos de $6^{\circ}$ grado a solicitar el material de figuras geométricas.

EJR: [El director responde] Dile al maestro JFHP que aquí están, pero que no se le olvide que a las 12:00 tenemos reunión de Enlace.

Niño: Sí, maestro (O.EJR.170412).

Ante esta dinámica de disciplina y obediencia donde se mueven los actores educativos, los cambios son difíciles; porque resulta más fácil hacer lo que por años ha funcionado, que reencauzar el rumbo hacia un trabajo participativo, colaborativo y de diálogo, para enfrentar los desafíos de desarrollo personal, a través de aprender a hacer y aprender a aprender desde su propio contexto.

\section{Tiempos para la colegialidad}

Para tratar asuntos didáctico-pedagógicos, la normatividad del Consejo Técnico Consultivo (SEP, 1982) establece reuniones una vez al mes, después del horario de trabajo. Sin embargo, se observó que las reuniones se celebran una o dos veces por semana al inicio de la jornada laboral y se hacen en el horario del recreo; en estos encuentros se abordan asuntos como la organización cotidiana de la escuela, la evaluación del programa de Carrera Magisterial y otros asuntos imprevistos. Al respecto, Encinas (2011) plantea que "los profesores de los colectivos impulsan diversas acciones, a saber: reuniones en los tiempos destinados regularmente al recreo escolar o mediante la prolongación de éstos" (p. 5), para abordar situaciones inmediatas que requieren de un tiempo breve.

Con la Reforma Educativa de 2013, a través de los Lineamientos para la organización y funcionamiento de los Consejos Técnicos Escolares (SEP, 2013a), se establece un órgano colegiado para tomar y ejecutar decisiones enfocadas a mejorar la calidad del servicio educativo, fomentar el desarrollo profesional de personal docente y directivo, así como fortalecer la autonomía de gestión de la escuela, a través de una participación obligatoria de sus miembros, con días, horario y temáticas definidas por la autoridad educativa, esto con el fin de superar las situaciones de falta de espacio y tiempo. A pesar de ello, en las escuelas se continúan realizando reuniones del profesorado dentro del tiempo de clase del alumnado.

En las entrevistas con los directores y directoras de educación primaria, la mayor parte coincide en señalar que las reuniones de trabajo colegiado las llevan a cabo en el tiempo de recreo de sus estudiantes, se trata de encuentros informales para tratar sobre todo asuntos urgentes, imprevistos o de organización, ya que el personal docente no está dispuesto a reunirse en horario fuera de la jornada laboral. Al respecto, tenemos la siguiente cita: 
EMC: ¿En qué momento de la jornada laboral desarrolla el trabajo colegiado en la escuela?

SCA: Durante la hora del recreo y al final del día (E.SCA.300812).

MC: ¿En qué espacio y tiempo convoca a colegiar a la planta docente?

LRP: De preferencia en los espacios de recreo, robamos unos minutos más para poder aterrizar los trabajos (E.LRP.290812).

En ocasiones, cuando se requiere de más tiempo, las reuniones formales del Consejo Técnico Consultivo se realizan después del recreo, una o dos veces al mes. El día que el personal docente se reúne, el alumnado es enviado temprano a casa, lo que genera inconformidad y malestar entre las familias, porque consideran que la continua suspensión de clases afecta el aprendizaje de sus hijos e hijas. Es así como identificamos:

La brecha entre el discurso y la realidad, mientras que en la retórica se destaca la importancia de que los profesores colaboren, en la práctica no se sabe bien ni cómo ni para qué deben hacerlo. Además, no existen condiciones (tiempos y espacios creados ex profeso para esta tarea) para la colegialidad docente, lo que revela el escaso valor que parece tener para las autoridades y para la propia institución (Moreno, 2006, p. 126).

A pesar de ello, la autoridad educativa utiliza las reuniones de Consejo Técnico para implementar proyectos, informar e instrumentar disposiciones que aseguren el óptimo funcionamiento de la escuela.

\section{Reflexiones finales}

La categoría de análisis empírica denominada "la colegialidad docente en la escuela primaria mexicana: algunas prácticas que la representan", la identificamos en un contexto sociocultural concreto, donde tanto la finalidad como los actores participantes, así como el espacio y tiempo de reunión para colegiar están vinculados a una visión de corto plazo. Los propósitos que motivan la colegialidad se enfocan en resolver situaciones inmediatas que surgen en el día a día. A menudo los sujetos participantes emplean principalmente una comunicación vertical $y$, en escasas ocasiones, formas más colaborativas, sobre todo, en el tiempo dedicado a la enseñanza y el aprendizaje o el recreo.

La colegialidad docente en la escuela primaria se desarrolla básicamente a partir de tres situaciones. La primera tiene como punto de partida los problemas cotidianos que surgen en el desarrollo del ejercicio docente, con lo cual, se favorece el diálogo entre el personal directivo, 
doi: http://dx.doi.org/10.15359/ree.21-2.6

URL: http://www.una.ac.cr/educare

CORREO: educare@una.cr

profesorado, familiay estudiantes, para identificar el origen, acordarlos mecanismosyalternativas para superarlos. Estas situaciones movilizan conocimientos, habilidades y actitudes que, según Perrenoud (2011), favorecen la competencia en situaciones complejas y difíciles, para hacer frente a lo inmediato y satisfacer los elementos básicos del conocimiento que permiten vivir y sobrevivir en el campo laboral. Al respecto, Hargreaves et al. (2008) aseguran que compartir ideas, conocimientos, experiencias e interactuar en grupo favorece el aprendizaje y se influye en él. De manera similar, Wenger (2011) afirma que aprender no se limita al contexto educativo, más bien se aprende toda la vida. En otras palabras, aprender es un proceso permanente y continuo que facilita la interacción y negociación de significados entre el profesorado.

En una segunda situación, aparece un escenario de reuniones cotidianas para brindar información sobre disposiciones y asuntos relacionados con la organización escolar, controladas por el director o directora, lo cual reafirma su autoridad ante el personal docente. Incluso, se pudo observar que en ocasiones el director o directora utiliza medios coercitivos como: notas de extrañamiento, disminución del puntaje de escalafón y malas notas en Carrera Magisterial, para forzar la colaboración y conservar la obediencia. Al respecto, Hargreaves (2005) señala que con ello se genera una colegialidad artificial impulsada y promovida por la autoridad educativa. Por un lado, se convoca a los maestros y maestras a colaborar y a trabajar en equipo; y por otro, se mantiene el control y la dirección del grupo. Situación que, evidentemente, atenta contra el cambio y la mejora de la educación.

En una tercera situación están las reuniones del director o directora con el personal docente, generadas por necesidades de conocimiento e información propias del quehacer profesional, tales como: situaciones relacionadas con la enseñanza y el aprendizaje, con asuntos de corte administrativo y con cambios en el plan y programas de estudio; lo que favorece, como afirman Stoll et al. (2004), procesos de investigación, reflexión, relación y la asunción de riesgos que movilicen el deseo de aprender. Ello supera el planteamiento de la colegialidad artificial referido por Hargreaves (2005) para dar paso a procesos de diálogo entre iguales, generando así conexiones para el aprendizaje. En consecuencia, Wenger (2011) señala que se puede llegar a la conformación de comunidades de aprendizaje, a partir de considerar compromisos mutuos, repertorios compartidos y una empresa conjunta (educación) para facilitar la negociación de significados entre acuerdos y desacuerdos, abundancias y carencias, alianzas y oposiciones, facilidad y esfuerzo, autoritarismo y participación, resistencia y conformidad, que finalmente consoliden un equipo con vocación de aprender. Consideramos que lograr esto requiere un cambio profundo en la cultura escolar de la escuela primaria, exige pasar de una cultura del conservadurismo, caracterizada por el individualismo y el ejercicio autoritaro del poder, hacia una cultura de la colaboración, permeable al cambio y la innovación, donde las relaciones entre los individuos sean horizontales y de apoyo mutuo. Sin duda, este nuevo escenario representa un serio desafío para todos los entes involucrados. 


\section{Referencias}

Castoriadis, C. (1997). El avance de la insignificancia. Buenos Aires: Eudeba.

De Jesús, J., De la Rosa, M. A. y García, G. (2003). El trabajo colegiado como una propuesta para la formación permanente de los docentes en la ENEP Acatlán. En Seminario de diagnóstico local. Recuperado de http://congreso.unam.mx/ponsemloc/ponencias/1392.html

Dirección General de Bachillerato. (2007). Consideraciones para el trabajo colegiado en academias. México. Recuperado de http://www.bachverdiu.com/content/ACADEMIASJULIO08/ COLEGIADOS.pdf

Encinas, A. (2011). El colectivo docente una comunidad de práctica situada y negociada. En XI Congreso Nacional de Investigación Educativa (pp. 1-9). México: Consejo Mexicano de investigación Educativa. Recuperado de http://www.comie.org.mx/congreso/ memoriaelectronica/v11/docs/area 15/0728.pdf

Fullan, M. (2002). Los nuevos significados del cambio en la educación. Barcelona: Octaedro.

Fullan, M. y Hargreaves, A. (1999). La escuela que queremos. Los objetivos por los que vale la pena luchar. México: SEP/Amorrortu.

García, N. (2005). La globalización imaginada. Buenos Aires: Paidós.

Gimeno, J. (2002). Educar y convivir en la cultura global. Las exigencias de la ciudadanía. Madrid: Morata.

Habermas, J. (2006). Teoría de la acción comunicativa I. Racionalidad de la acción y racionalidad social ( $2^{a}$ reimpresión). Madrid: Taurus.

Hargreaves, A. (2005). Profesorado, cultura y postmodernidad. (Cambian los tiempos, cambia el profesorado) (5a ed.). Madrid: Morata.

Hargreaves, A., Earl, L. y Ryan, J. (2008). Una educación para el cambio. Reinventar la educación de los adolescentes. Barcelona: Octaedro.

Heller, A. (1996). Una revisión de la teoría de las necesidades. Barcelona: Paidós. Recuperado de https://www.academia.edu/9956257/Una revisi\%C3\%B3n de las teor\%C3\%ADas de las necesidades

Lobato, O. y De la Garza, E. (2009). La organización del cuerpo académico: Las premisas de decisión, colegialidad y respuesta grupal. Un acercamiento desde su autorreferencialidad. Estudio de caso comparativo en la educación de la ingeniería. Revista Mexicana de Investigación Educativa, 14(40), 191-216. Recuperado de http://www.scielo.org.mx/pdf/ rmie/v14n40/v14n40a10.pdf 
doi: http://dx.doi.org/10.15359/ree.21-2.6

URL: http://www.una.ac.cr/educare

CORREO: educare@una.cr

Lugo, E. (2004). Los desafíos de la UAEM para transitar hacia una institución colegiada. Congreso retos y expectativas de la universidad. $3^{\circ}$ Nacional y $2^{\circ}$ Internacional (pp. 1-15). México: ANUIES. Recuperado de https://www.yumpu.com/es/document/view/14538751/titulolos-desafios-de-la-uaem-para-transitar-hacia-una-institucion-

Martínez-Cruz, E. (2013). El papel del directoren el contextoeducativo:La evaluación ylacolegialidad como exigencias de la escuela primaria. El caso de la zona escolar N. 127 (Tesis doctoral). Universidad Autónoma del Estado de Hidalgo, México, Pachuca.

Moreno, T. (2006). La colaboración y la colegialidad docente en la universidad: Del discurso a la realidad. Perfiles Educativos, 28(112), 98-130. Recuperado de http://www.scielo.org.mx/ pdf/peredu/v28n112/n112a5.pdf

Morin, E. (1999). Los siete saberes necesarios para la educación del futuro. París: Unesco.

Organización de las Naciones Unidas (ONU). (2000). Cumbre milenio 2000. Declaración del milenio de las Naciones Unidas. Objetivos de desarrollo del milenio. Recuperado de http://www. un.org/es/events/pastevents/millennium summit/

Pérez, Á. I. (2004). La cultura escolar en la sociedad neoliberal (4a ed.). Madrid: Morata.

Perrenoud, P. (2011). Construir competencias desde la escuela. México: J. C. Sáez editor.

Pujadas, C. y Durand, J. (2002). El concepto ampliado de colegialidad: Alcance y posibilidades. Fundamentos en humanidades, 3(5-6), 57-68. Recuperado de http://www.redalyc.org/ articulo.oa?id=18400504

Sánchez, M. A., Nishikawa, A. K., Cordero, G. y Bocanegra, N. (2008). La colegialidad invisible en la educación básica. Perfiles Educativos, 30(119), 55-71. Recuperado de http://www.scielo. org.mx/pdf/peredu/v30n119/v30n119a4.pdf

Secretaría de Educación Pública (SEP). (7 de diciembre, 1982). Acuerdo 96, que establece la organización y funcionamiento de las escuelas primarias. Diario Oficial de la Federación. México.

Secretaría de Educación Pública (SEP). (19 de mayo, 1992). Acuerdo nacional para la modernización de la educación básica. Diario Oficial de la Federación. México. Recuperado de https://www.sep.gob.mx/work/models/sep1/Resource/b490561c-5c33-4254-ad1caad33765928a/07104.pdf

Secretaría de Educación Pública (SEP). (1999). Propuesta para el desarrollo del trabajo colegiado en las escuelas normales. México. Recuperado de http://www.normalsuperior.com.mx/ ens/files/escolarizada/TRABAJO COLEGIADO.pdf 
Secretaría de Educación Pública (SEP). (2001). Programa nacional deeducación 2001-2006. México: Autor. Recuperado de www.oei.es/historico/quipu/mexico/Plan educ 2001 2006.pdf

Secretaría de Educación Pública (SEP). (2007). Programa sectorial de educación 2007-2012. México: Autor. Recuperado de https://coleccion.siaeducacion.org/sites/default/files/ programa sectorial educacion mexico.pdf

Secretaría de Educación Pública (SEP). (2013a). Lineamientos para la organización y funcionamiento de los consejos técnicos escolares. México: Autor. Recuperado de http:// es.slideshare.net/Valfh/lineamientos-consejo-tecnico-escolar

Secretaría de Educación Pública (SEP). (2013b). Programa sectorial de educación 2013-2018. México: Autor. Recuperado de https://coleccion.siaeducacion.org/sites/default/files/files/ programa sectorial de educacion 20132018 web.pdf

Secretaría de Educación Pública (SEP). (s. f.). Lineamientos de trabajo colegiado. México: Autor. Recuperado de http://www.dgb.sep.gob.mx/informacion-academica/otros/ltrabajocolegiado.pdf

Stoll, L., Fink, D. y Earl, L. (2004). Sobre el aprender y el tiempo que requiere. Implicaciones para la escuela. Barcelona: Octaedro.

Unesco. (2000). Foro Mundial sobre la Educación. Marcos de acción de Dakar. Educación para todos: Cumplir nuestros compromisos comunes. Dakar, Senegal. Francia: Autor. Recuperado de http://unesdoc.unesco.org/images/0012/001211/121147s.pdf

WCEFA. (1990). Satisfacción de las necesidades básicas de aprendizaje. Una visión para el decenio de 1990. Conferencia Mundial sobre educación para todos. Jomtien, Tailandia. Nueva York: Autor. Recuperado de http://unesdoc.unesco.org/images/0009/000975/097552SB.pdf

Wenger, E. (2011). Comunidad de práctica. Aprendizaje, significado e identidad. Barcelona: Paidós. 